Original Research Paper

\title{
Adaptive and Neural Network Based Control of Pitch of Unmanned Aerial Vehicles
}

\author{
Mackenzie T. Matthews and Sun Yi \\ Department of Mechanical Engineering, North Carolina A\&T State University, Greensboro, USA
}

\author{
Article history \\ Received: 03-10-2019 \\ Revised: 24-10-2019 \\ Accepted: 12-11-2019 \\ Corresponding Author: \\ Mackenzie T. Matthews \\ Department of Mechanical \\ Engineering, North Carolina \\ A\&T State University, \\ Greensboro, USA \\ E-mail: mtmatthe@aggies.ncat.edu
}

\section{Introduction}

Effective control of a quadrotor type of Unmanned Aerial Vehicles (UAVs) have been the main focus of literature during recent years. These control systems are used for carrying out activities such as law enforcement surveillance, agricultural maintenance, acquiring movie and sports event footage (Horsman, 2016). Moreover, UAVs are extremely important in various missions, such as military operations, search and rescue and serving as personal assistants (Armah, 2018). One of the models for an unmanned aerial vehicle is the AR Drone 2.0, which is shown in Fig. 1 (Matthews and Yi, 2019). The unmanned aerial

\begin{abstract}
The objective is to develop a new control strategy for quadrotor types of Unmanned Aerial Systems (UAVs) that assist in closing the existing research gaps between the undesired uncertainties and current control systems. This research investigates a system modeling and the effectiveness of error elimination through adaptation in the change of the plant/system's output. The UAV is controlled via Wi-Fi using MATLAB. Proportional-Integral-Derivative (PID) controller, the two degrees of freedom Proportional-Integral-Derivative (2DOF-PID) controller and the model reference Neural Network (NN) controller are used for the aircraft pitch control. External disturbances and modeling errors can lead to the instability and unpredictable behaviors of a system. Even though the redurbances cannot be avoided the effects of such disturbances can be high-performance tracking in the presence of the disturbances. The robust control systems with the adaptive control will improve navigation performance and operation for military, reconnaissance and surveillance applications. The application of a robust controller determines the performance by observing the system's behavior to identify and reduce the unpredictable effects of disturbances and achieve a better behavior of the whole system. By observing the system, the neural network is suitably used to learn from a set of training patterns. System identification uses a neural network to capture the behavior of system dynamics, which assists the neural network to train itself to act as a controller. Moreover, the demonstrate the effectiveness in improving the speed and stability for the dynamic system. The presented work will produce a flexible, robust and effective control system model that provides additional stability and reduce the effects of disturbances.
\end{abstract}

Keywords: UAV, PID, 2DOF-PID, Model Reference Adaptive Control (MRAC), Neural Network (NN) vehicle systems have been used for military applications, and its technology and capabilities are evolving and expanding for more onboard intelligence and reconnaissance data applications.

The focus of the research presented is the improvement of the control models with new compensators that provide a better and efficient aerodynamic structure for acute navigation and sensing operation for unmanned aerial vehicle systems. Moreover, the developed robust control systems will improve the reliability and stability of the unmanned aerial vehicle systems for military, reconnaissance and surveillance applications. The unmanned aerial vehicle 
used for this study is the Aerosonde UAV System with six degrees freedom (Socas et al., 2015). The nonlinear model is described with 12 dynamic variables: body frame velocities $(u, v, w)$, Euler angles, $(\varphi, \theta, \psi)$ angular velocities $(p, q, r)$ and inertial positions $\left(p_{N}, p_{E}, h\right)$. Consequently, the model depends on external forces $\left(f_{x}, f_{y}, f_{z}\right)$ and moments $(l, m, n)$ (Sarhan and Qin, 2016). The applications of this specific drone system provide intelligence, surveillance, reconnaissance, communications relay and other applications in a single flight. The Aerosonde UAV has a long and effective history of performance in very harsh environments.

The MATLAB/Simulink model provides a system by constructing mathematical models of dynamic systems from a measured input-output data (Armah, 2015). The model system is a set of interacting components subjected to various inputs and produces outputs to perform a specific function. The input of a model system affects the dynamics of the system and the output attributes the dynamics of the system. Moreover, the mathematical model has a relationship between the inputs and outputs of the system. The mathematical description of the model presents the relation between the inputs and outputs of the system and the connection to other systems. Whereas, it has an approximation of the real physical system to be controlled. The accuracy of a model depends on the specific needs and the use of the equation of motion that represents the mathematical description of the model. The control's objective defines the parameters to achieve the goal of a response of the system. These parameters are obtained using MATLAB and through numerical simulations. The feedback closed control loop that controls the system represents three concepts for a model system, which measure, compare and adjust the model system. The results will obtain a healthy, robust and well-functioning system, which use the neural network to optimize the performance and to achieve a better behavior response of the system.

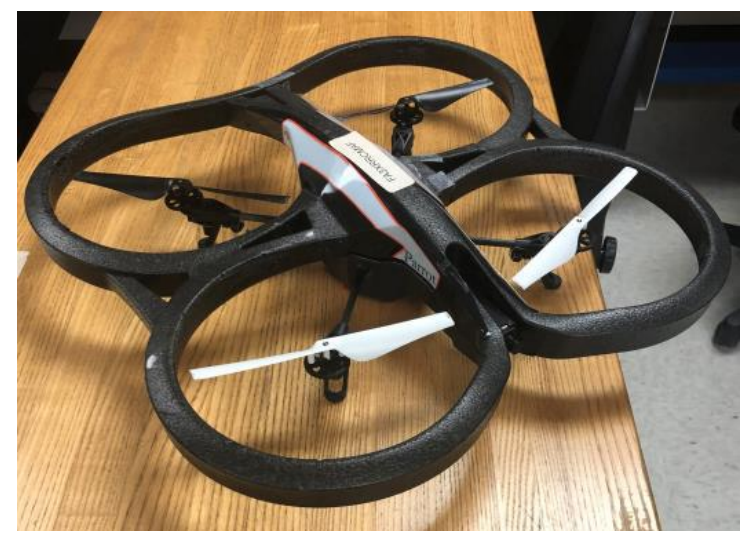

Fig. 1: Unmanned aerial vehicle
This research will promote new ways of understanding the MRAC direct method approach to a high order adaptive control with the NN controller, the 2DOF-PID controller and the PID controller. Likewise, the simulation model of the UAV uses gamma and alpha values in the adaptive mechanism model for each specific controller. The NN controller performs plant identification and control design. The PID and 2DOF-PID controllers with traditional fixed parameters direct the plant with an applied disturbance to obtain the best performance analysis and robustness to external disturbances and model parametric uncertainty. This scope of work will produce a flexible, robust and effective control system model that provides additional stability and reduces the effects of disturbances. Moreover, the main component develops a robust control system and improves the reliability and stability of the UAV.

\section{Literature Review}

In the proposed research by Armah (2018), a tuned proportional-plus velocity combined with the Model Reference Adaptive Control (PV-MRAC) evaluates quadrotor types of UAVs which considers time delays in altitude control system (Armah, 2018). For research consideration, Eressa et al. (2016) observe the performance difference and similarity in model system dynamics and the observation of the efficiency of error elimination with less fluctuation and adaptability to set point variation between two controllers (Eressa et al., 2016). The controllers were traditional fixed parameter PID controller and the model reference $\mathrm{NN}$ controller acquires new knowledge to advance new methods of understanding.

In Sarhan and Qin's research study, the adaptive PID control of UAV altitude dynamics is built on parameter optimization with fuzzy inference. Likewise, the proposed adaptive PID control is a combination of traditional PID and fuzzy logic control schemes (Sarhan and Qin, 2016). A simple adaptive control scheme based on Model Reference Adaptive Systems (MRAS) algorithm is developed for the asymptotic output tracking problems with changing system parameters and disturbances under guaranteeing stability, in which the adaptive adjusting law is derived by using the Lyapunov Theory (Cuong et al., 2013).

According to Goel et al. (2016), it compares the MRAC and Modified MRAC scheme when the adaptation laws are designed using different approaches. Whereas, the controller parameter adaptation laws are designed based on the MIT Rule and Lyapunov Theory (Goel et al., 2016). According to Jain and Nigam (2013), the design of a controller for a second order system using the MRAC scheme uses the adaptive mechanism MIT Rule with the standardized algorithm to manage the changes in the reference signal. Therefore, this 
adaptation law known to as the Modified MIT Rule (Jain and Nigam, 2013). Swarnkar et al. (2011a) discuss the application of model reference adaptive control scheme and the system performance compares the Lyapunov Rule and MIT Rule. The comparison was performed with different values of adaptation gain between two rules (Swarnkar et al., 2011a). The research in Swarnkar et al. (2011b) deals with the implementation of the MRAC system to second order models with varying adaptation gain values for which the MIT Rule is applied (Swarnkar et al., 2011b).

In Dydek et al. (2013) research, the study investigates the approach of the application of direct and indirect model reference adaptive control to a lightweight low-cost quadrotor unmanned aerial vehicle platform. Whereas, the design of the adaptive controller is presented and followed by a comparison of flight test results using the existing linear and augmented adaptive controllers (Dydek et al., 2013). Ghaffar and Richardson report that a model reference adaptive control and a fixed gain LQR control were implemented in the height controller of a quadrotor that has parametric uncertainties due to the act of picking up an object of unknown dimension and mass (Ghaffar and Richardson, 2015). Therefore, in the presence of parametric uncertainties, the combination of adaptive and fixed gain control in the controller architecture can lead to improved monitoring results (Ghaffar and Richardson, 2015).

\section{Methodology}

\section{Modeling}

The importance of this model study is to enhance the UAV model with a high order of adaptive control to accommodate uncertainties. This will provide an improved and efficient aerodynamic structure for acute navigation and sensing operation for the fixed-wing UAV system. According to Eressa, the study applies the equation of motion only in the pitch direction. The pitch is in an upward direction, instead of the downward direction. The differential equation used is expressed as (Eressa et al., 2016):

$$
\ddot{x}_{\theta}=-0.4988 \dot{x}_{\theta}-13.8636 x_{\theta}+18.2386 \delta_{e}
$$

The Equation 1 is transformed into the Laplace domain as:

$$
s^{2} x_{\theta}(s)=-0.4988 s x_{\theta}(s)-13.8636 x_{\theta}(s)+18.2386 \delta_{e}(s)
$$

Equation 1 represents the longitudinal dynamics of the UAV, which is simplified from the $5^{\text {th }}$ order to a $2^{\text {nd }}$ order approximated transfer function. In addition, the focus is to find a minimized order transfer function relating the elevator deflection $\delta_{e}$ to pitch angle $x_{\theta}$. The initial condition is $x(0)=0$ Therefore, a short period approximation is used. The input $u(s)$ correlates with the elevator deflection $\delta_{e}(s)$ and $y(s)$ correlates with the pitch angle $x_{\theta}(s)$, which is the measured output signal. in According to Eressa, work, the transfer function relates with the pitch angle $x_{\theta}(s)$ to the elevator deflection $\delta_{e}(s)$, which can be written in transfer form as (Eressa et al., 2016):

$G(s)=\frac{x_{\theta}(s)}{\delta_{e}(s)}=\frac{18.2386}{s^{2}+0.4988 s+13.8636}$

Transfer function in Equation 3 can be converted into the State-Space Matrix-Vector Form below:

$$
\begin{aligned}
& \dot{x}(t)=\left[\begin{array}{cc}
-0.4988 & -13.8636 \\
1.0000 & 0
\end{array}\right] x(t)+\left[\begin{array}{l}
1 \\
0
\end{array}\right] u(t) \\
& y(t)=\left[\begin{array}{ll}
0 & 18.2386
\end{array}\right] x(t)+[0] u(t)
\end{aligned}
$$

In the matrices of Equation 4 and Equation 5, the rank of both controllability and observability matrices is 2 , which means it is controllable and observable. The disturbance can lead to the instability and uncertainty behaviors of a system. In addition, a disturbance is set to 1 and is applied to the State-Space Matrix-Vector. The state space equation with a disturbance represents the plant/system of the UAV, which achieves a desired response of the UAV system.

In Fig. 2, the plant is part of the model which is a mathematically complex that describes the system. It resembles the system itself and it represents the receiver and activates the signal. When the controller sends out the signal to define the behavior of the output and sets the connected parts of the system with the input and output variables. The role of the plant is to apply the dynamics of the output and for the system to be controlled, which displays a nonlinear and constant behavior. In addition, the controller sends the information to the plant and the plant activates or reacts on the information. Whereas, the new output is obtained. The input of the plant causes the measured values to track the desired values. The plant location demonstrates the output of the controller with the input of the plant. A plant's description is controlled as the controller's input (Ioannou and Baldi, 2010). The output of the plant is the reference signal, which generates the desired output of the system. This is why the plant is used to follow the model of the system in a precise manner as possible. The transfer function and state space vector form are examples of a plant, which can use the measured values for an UAV. 


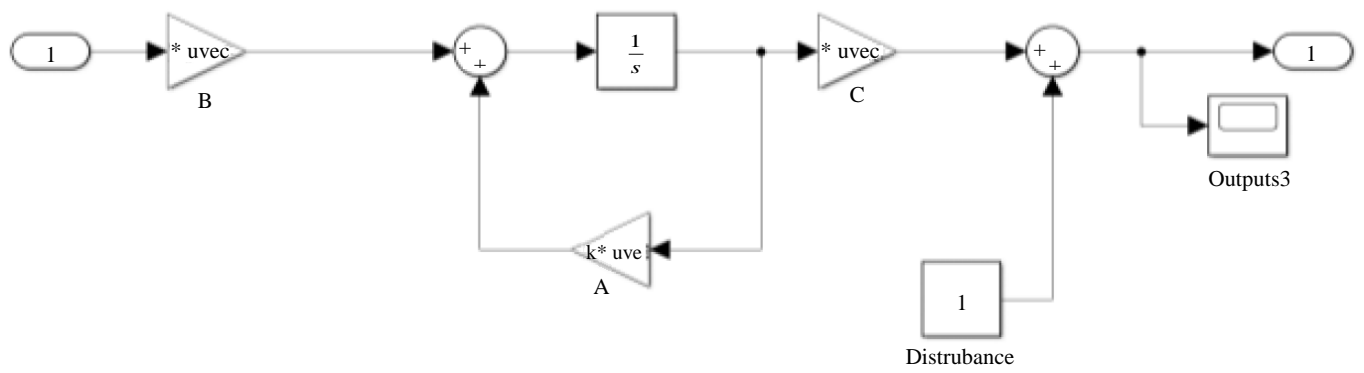

Fig. 2: Plant with disturbance

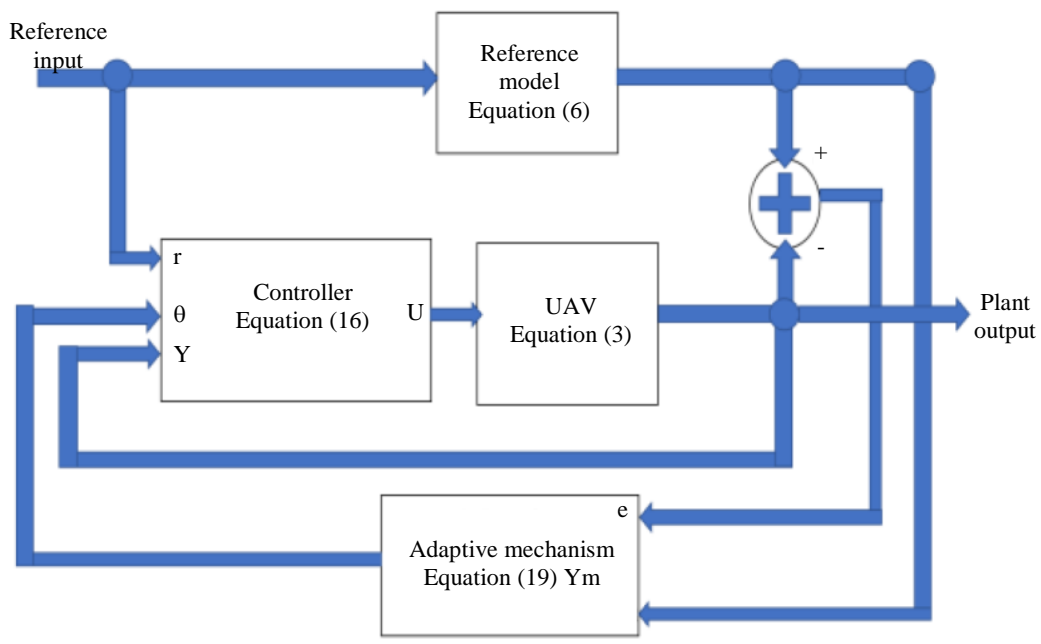

Fig. 3: Model Reference Adaptive Controller (MRAC)

\section{Simulation}

In Fig. 3, three main elements of this model are the Reference Model, the Plant Model and the Adaptive Controller. It demonstrates the design and model adaptive controller using Simulink. Likewise, the Adaptive Controller has two subcomponents, which are the controller and the adaptive mechanism.

\section{Design of Control}

With the implementation of the different gamma values, the adaptation gain $(\gamma)$, changes the learning rate and reduces the error between the plant and reference model outputs. Whereas, all the signals in the closed-loop plant are bound. The plant output tracks the desired set point as close as possible. Likewise, the adaptive control accommodates and includes the parametric uncertainties for the UAV. As $\alpha, \zeta$ and $w_{n}$ are represented as zeta and natural frequency. They are set to $1,0.8$ and $4.236 \mathrm{rad} / \mathrm{sec}$, which receives suitable performances for both settling time and actuator effort (Eressa et al., 2016). Thus, the $\alpha, \zeta$ and $w_{n}$ are used to determine the important values for the reference model and the values of the
PID controllers. In this research approach, the main components of this model structure are expressed as follows:

\section{Reference Model}

A model can be defined as the reference model of a system that proceeds to function as an individual model and prevails in an entire parent model. The reference model determines a desired trajectory that the process output should follow for a given change in the set-point (Kasparian and Batur, 1998). It creates the plant's output of the system to accomplish the desired response of the system. The reference model is designed for a given reference input signal and the output of the reference model represents the desired response the plant output should follow (Ioannou and Baldi, 2010). Specifically, the output of the system should relate to feedback from a reference model. This shapes the desired values that combine into adaptable limitations to generate the plant feedback to equal the feedback of the reference model. It utilizes the reference model in selecting a plant model in which the description and dynamics are well known in order to create the neural networks. 
For the reference model, the natural frequency and zeta determine the transfer function. The reference model displays a transient response, which is a second order system transfer function used as the desired response. The equation for natural frequency and zeta are expressed as:

$$
y=\frac{w_{n}^{2}}{s^{2}+2 \zeta w_{n} s+w_{n}^{2}} u
$$

Where:

$$
\begin{aligned}
& w_{n}=\text { The natural frequency }(\mathrm{rad} / \mathrm{sec}) \\
& \zeta=\text { The damping ratio (zeta) } \\
& u=\text { The control signal } \\
& y=\text { The measured signal output }
\end{aligned}
$$

Equation 6 correlates with Fig. 4, which displays the values implemented in the reference model block. Moreover, the reference model is an input and a random reference of the PID, 2DOF-PID and NN controller, which produces the actual response on the system. Hence, the NN optimizes the input of the plant's behavior response of the system. In other words, the $\mathrm{NN}$ demonstrates a different response from the actual response.

\section{Controller}

The controller is created and it is defined by a set of adjustable parameters for the plant system, the general function of the controller maintains the controlled variable near its desired value when these parameters occur (Palm, 2014). The design of a controller can alter or modify the behavior and the response of an unknown plant to meet certain performance requirements are tedious and a challenging problem in many control applications (Ioannou and Baldi, 2010). The controller is designed to meet the performance requirements for the plant and sends the information to the plant (Ioannou and Baldi, 2010). In other words, a good controller makes a good model of the system. Hence, the controller can be applied in an open loop system and closed loop system. The plant is the output of the controller and the input of the controller is the reference signal. The importance of the controller is to perform the transformation (Janert, 2013). For the 2DOF-PID controller, the controller is implemented by using the approach introduced in Eressa et al. (2016). In Equation (6), it correlates with Fig. 3, which displays the values implemented in the reference model block. Input and Output equation of the two degrees of freedom PID controller is given:

$$
u(t)=k_{p} y(t)+k_{i} \int(r(t)-y(t))-k_{d} \dot{y}
$$

Where:

$k_{p}=$ The proportional gain

$k_{i}=$ The integrator gain $k_{d}=$ The derivative gain

$t=$ The time $(\mathrm{sec})$

$r=$ The reference desired signal input

The desired closed loop response is used to determine the PID parameters with $\alpha, \zeta$ and $w_{n}$, which are the free parameters shown as:

$$
y(s)=\alpha w_{n} \frac{w_{n}^{2}}{\left(s+\alpha w_{n}\right)\left(s^{2}+s 2 \zeta w_{n} s+w_{n}^{2}\right)}
$$

This particular $\alpha$ is a derivative filter constant (Alfaro and Vilanova, 2016). In the Eressa et al. (2016) paper, the characteristic equation is used to determine the PID parameters (Eressa et al., 2016). It uses three mathematical control functions and applies them to input signals for desired outputs (Aamir, 2013). Proportional term value determines the response of the system towards current error (Aamir, 2013). Integral term value fastens the response and reduces the error. Thus, the derivative part reduces overshoots and oscillations. For the PID controller, it is simple and robust over a wide range of operating conditions. It has easy adjustment and high reliability (Cuong et al., 2013). It is often the first choice for a new controller design (Cuong et al., 2013). The development of the PID controller design has advanced from mechanical devices to digital devices, but the control algorithm is almost the same (Cuong et al., 2013). In the time domain, the Input and Output equation of the PID controller is given:

$u(t)=k_{p} e(t)+k_{i} \int e(t) d t+k_{d} / d e / d t$

In the Laplace Transform of the s-domain, the equation of the PID controller is represented as:

$U(s)=K_{p} E(s)+K_{i} \frac{1}{s} E(s)+K_{d} s E(s)$

In addition, $E(s)=Y(s)-U(s)$ and the controller is equivalent to the equation given as:

$U(s)=C(s) E(s)$

where, $G(\mathrm{~s})$ is the transfer function is given by Equation 3 and $C(s)$ is:

$C(s)=\frac{\left(k_{d} s^{2}+k_{p} s+k_{i}\right)}{s}$

The combined system of $G(s)$ and $C(s)$ are regular functions that act on the tracking error $E(s)$ to obtain the output $Y(s)$. The closed loop arrangement of the transfer function summarizes as: 


$$
Y(s)=\frac{C(s) * G(s)}{1+C(s) * G(s)} R(s)
$$

Substitute Equation 3 and Equation 12 into Equation 13 , the closed loop transfer function from $Y(s)$ to $R(s)$ is expressed as:

$$
\frac{Y(s)}{R(s)}=\frac{N(s)}{D(s)}
$$

The desired closed-loop response correlates with the reference model is used to determine the PID parameters with the $\zeta$ and $w_{n}$, which are the free parameters and shown in Equation 6. The PID parameters are determined by using the desired characteristic poles/roots, which are determined and expressed as:

$$
s=-\zeta^{*} W_{n} \pm W_{n} \sqrt{1-\zeta^{2} i}
$$

Since it is the third order in the closed-loop transfer function plant. It assumes to add another desired root/eigenvalue of -1 to help compare the characteristics $D(s)$ of the closed loop transfer function to the denominator of the reference model.

The NN Controller is one of the popular neural network architectures for prediction and control that was been implemented in the NN Toolbox ${ }^{\mathrm{TM}}$ software as shown in Fig. 6. The model reference controller is a neural network trained to control a plant to follow a reference model (Suzuki, 2011).

The neural model reference control architecture uses two neural networks: the controller network and the plant model network as shown in Fig. 5. Whereas, the plant model is identified first and the controller is trained for the plant output to follow the reference model output (Suzuki, 2011). The neural network estimates and predicts the output of the system while working with the plant. In other words, the neural network works with the plant of the model system and applies the exact same operations to the plant.

The neural network process model is needed to obtain an estimate of the sensitivity of the plant in regards to its inputs and provides the information needed for the plant (Kasparian and Batur, 1998). Therefore, a neural network is designed to identify the plant and learns the plant behavior through some form of training. In addition, the neural network acts as the controller, which develops and trains to understand the plant behavior. Hence, the plant of the system feedback traces the reference model.

Neural networks have been successfully used to model linear, nonlinear and inverse dynamics of systems (Kasparian and Batur, 1998). The neural network controller consists of two neural networks, which deals with the plant identification and control design to stabilize the system's response. The neural network predicts the output of the function by observing the behavior of the system and investigates the performance by observing the system's behavior. By observing the system's behavior, the neural network is suitably attained by a process of adaptation or learning from a set of training patterns.

Neural networks are suitable for modeling and function approximation purposes with the action. The two neural network architecture and data processing steps are comparable. However, the number of delayed inputs are different (Eressa et al., 2016). Whereas, $y[k$ $n]$ for $n=0,1,2$ are current/delayed plant outputs; $C[k$ $n$ ] for $n=0,1,2$ are current/delayed controller outputs; $u[k-n]$ for $n=0,1,2$ are delayed plant inputs; $r[k-n]$ for $n=0,1,2$ are delayed input reference; $f($.$) is a nonlinear$ mapping function (Eressa et al., 2016). The neural network controller's focus is to control the operation of the overall behavior of the reference model, which is trained by the plant.

\section{Adaptive Mechanism with Modified MIT Rule}

The PID, 2DOF-PID and NN controllers output are $U$, which operates with the adaptive mechanism output (theta) expressed as:

$\left\{\begin{array}{c}U=\text { Upid }^{*} \text { theta } \\ U=U 2 \text { dofpid } * \text { theta } \\ U=\text { Unn }^{*} \text { theta }\end{array}\right.$

The MIT Rule was first developed in 1960 by the researchers of Massachusetts Institute of Technology (MIT) and used to design the autopilot system for aircrafts (Jain and Nigam, 2013). According to the MIT Rule, the $(e)$ presents the error between the outputs of the plant and the reference model. Theta $(\theta)$ is the adjustable parameter. In the MIT Rule, a cost function is defined as:

$J(\theta)=e^{2} / 2$

Parameter $\theta$ represents the output that is adjusted in such a manner. Therefore, the cost function is minimized to zero based on the plant output $(Y)$ and reference model output $\left(Y_{m}\right)$. The change in the parameter is kept in the direction of the negative gradient of $J$ (Alfaro and Vilanova, 2016):

$\frac{\partial \theta}{\partial t}=-\gamma \frac{\partial J}{\partial \theta}=-\gamma e \frac{\partial e}{\partial \theta}$

Where:

$\frac{\partial e}{\partial \theta}=$ The sensitivity derivative of the system

$\theta=$ The unknown theta parameter output

$\gamma=$ The gamma value 


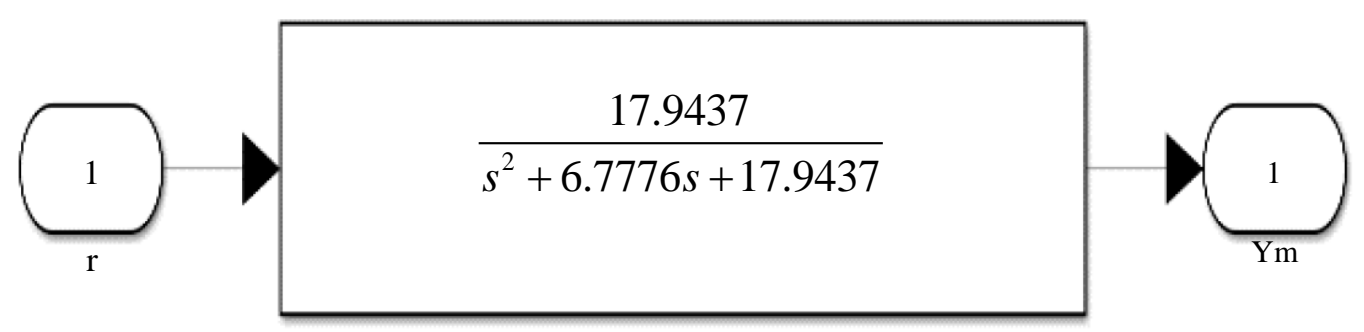

Fig. 4: Reference model

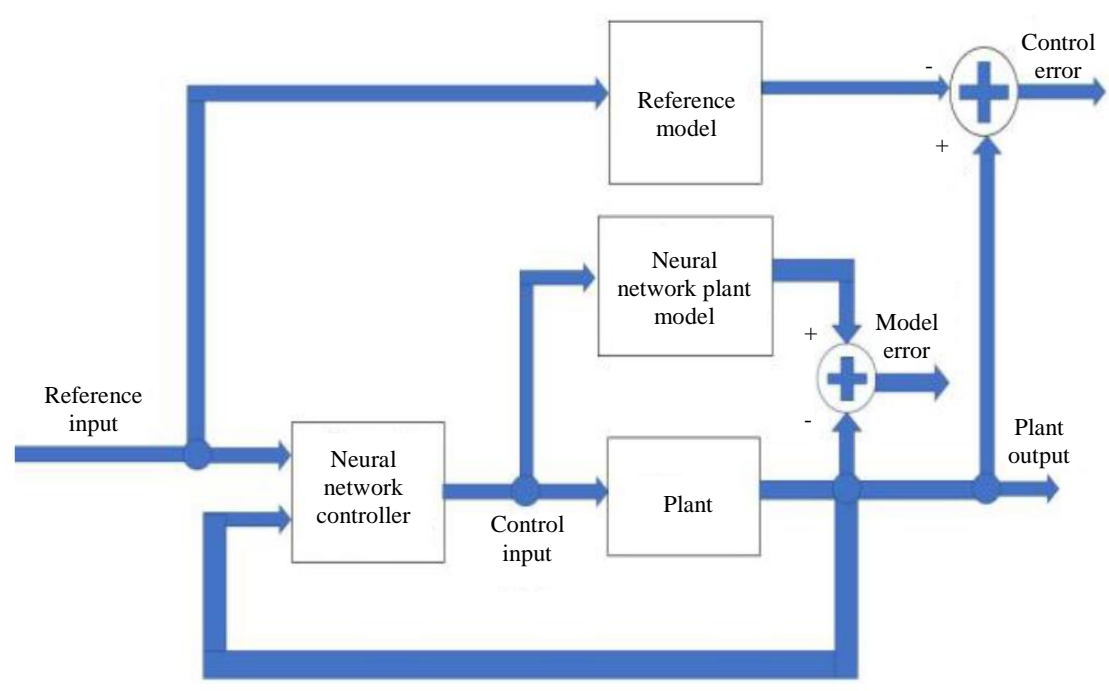

Fig. 5: Model reference neural network controller

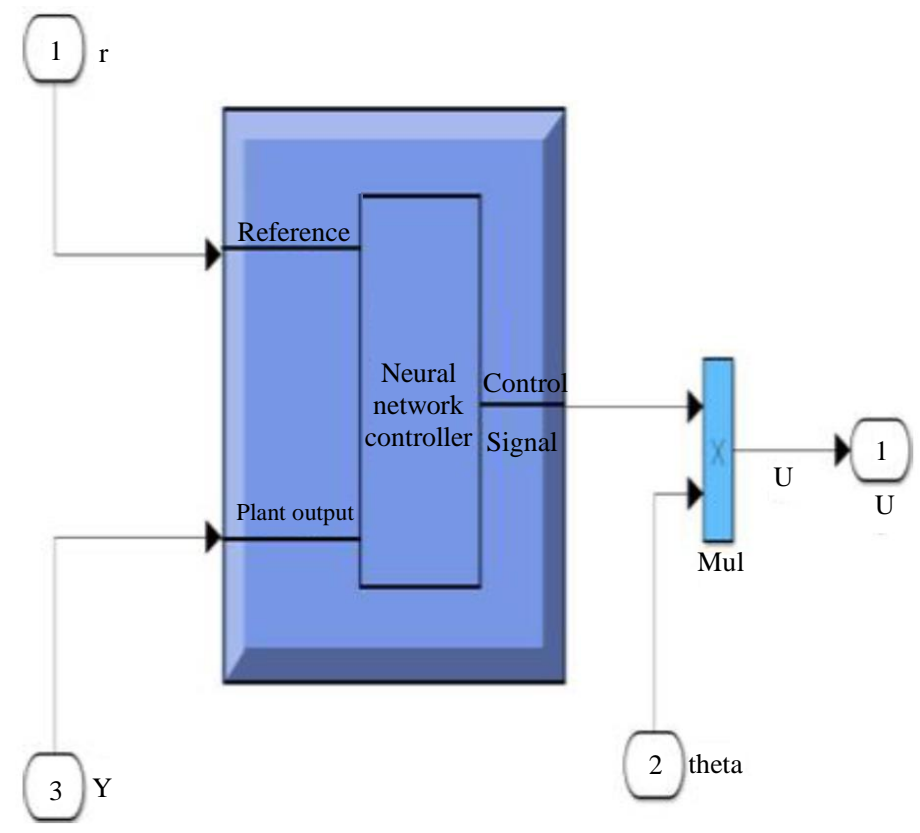

Fig. 6: Neural network controller 
The sensitivity derivative of the system demonstrates how the error is adjusting with the change in the parameter $\theta$ from Equation 18. It displays the change in the parameter $\theta$ with the change in time with the cost function $J(\theta)$ that will decrease to zero (Swathi and Ramesh, 2017). The gamma value is present as $\gamma / s$, which is used to see the system's effect and to produce a learning curve to minimize the error between the outputs of the plant and the reference model. The Modified MIT Rule can be used to design a controller for any system with a Modified MRAC scheme. In addition, it uses normalized algorithm to handle variations in the reference signal. Therefore, it is applied in the adaptation mechanism to be used with the MIT Rule to develop the modified control law, which works with the adaption gain to be modified in Fig. 7. The reason for the MRAC with the Modified MIT Rule is because the feedback controllers have some problems regarding the changes in the environmental conditions and the variation in the character of the disturbances.

In Fig. 7, it illustrates the adaptive mechanism model with a modified control law block. It adjusts the parameters in the control law. The Adaptation Law searches for the parameters that the response of the plant should be the same as the reference model. It is designed to guarantee the stability of the control system as well as conversance of tracking error to zero (Swarnkar et al., 2011b). The gamma value is present as $\gamma / s$, which is applied to see the effect of the system. The output of theta $(\theta)$ is predicated on the error $(e)$ between plant output $(Y)$ and reference model output $\left(Y_{m}\right)$.
The MIT Rule is very sensitive to changes in the amplitude of the reference input. The system may become unstable if it has large values of reference input. Thus, the use of the normalized algorithm is applied with the MIT Rule to create a control law to protect against dependence on the amplitude of the signal. With normalization, the MIT rule can make the plant follow the model as accurately as possible. The normalized algorithm modifies the adaption. The Adaption Law is expressed:

$$
\left\{\begin{array}{c}
\varphi=\frac{\partial e}{\partial \theta}=\frac{Y_{m}}{\theta_{o}} \\
\frac{\partial \theta}{\partial t}=\frac{\gamma e \varphi}{\alpha+\varphi^{\prime} \varphi}=\frac{\gamma e^{*}\left(\frac{Y_{m}}{\theta_{o}}\right)}{\alpha+\left(\frac{Y_{m}}{\theta_{o}}\right)^{2}}=\frac{\gamma e^{*} Y_{m}}{\alpha+Y_{m}{ }^{2}}
\end{array}\right.
$$

where, $\theta_{o}=$ known theta parameter output.

where, $\varphi=\frac{\partial e}{\partial \theta}$ and $\alpha(\alpha>0)$ is introduced to remove the difficulty of zero division when $\varphi$ is small (Jain and Nigam, 2013). Take into consideration, the gamma and alpha are set to 0.5 and 0.1 . The NN controller acts as the controller to direct the plant, which is one of the components for the MRAC. In addition, it operates with the adaptive mechanism output (theta) that is used to define the controller law. The value of $\theta$ is primarily dependent on adaptation gain (Jain and Nigam, 2013).

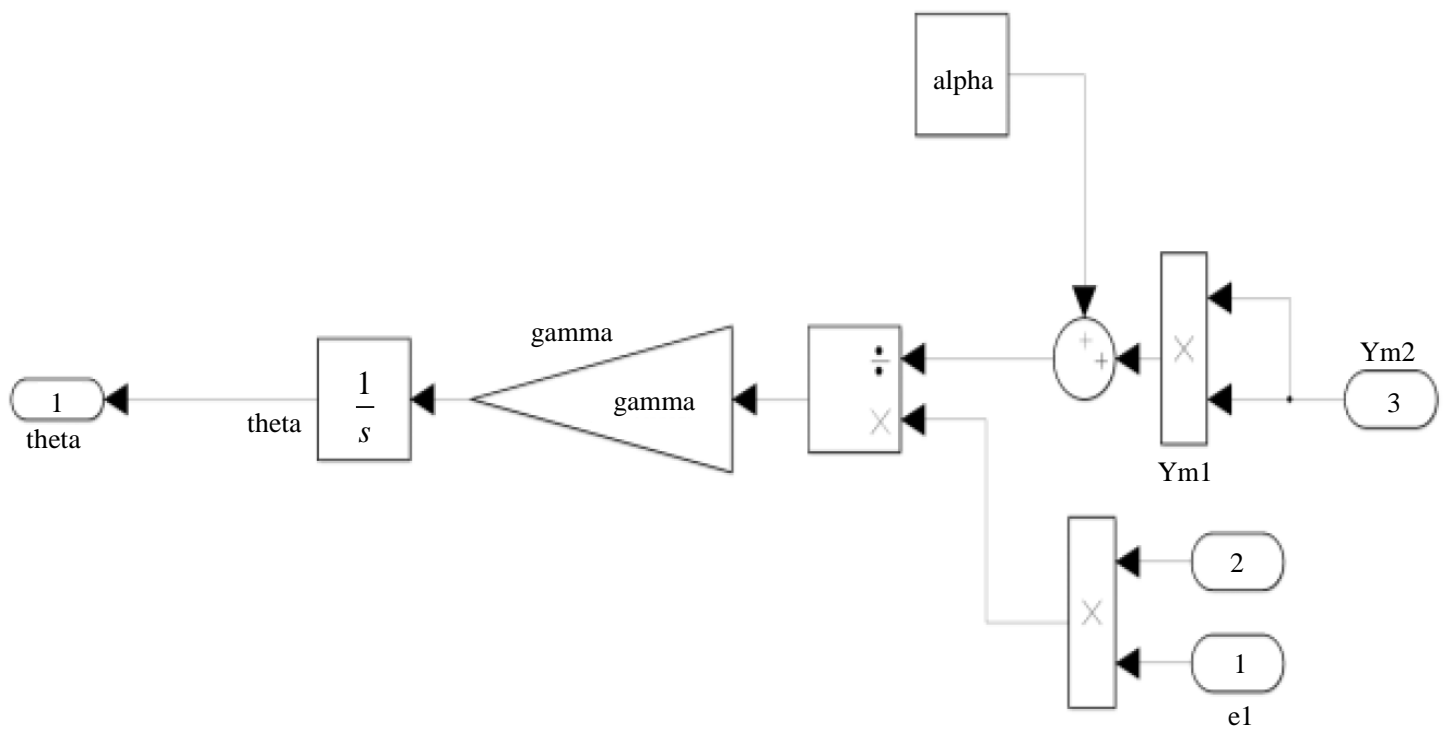

Fig. 7: Adaptive Mechanism with Modified MIT Rule 


\section{Validation and Observation}

The simulation results are produced in MATLAB for analysis of control schemes for the pitch direction response of a UAV given in the pulse generator's response. The performance is measured in terms of rise time $\left(T_{r}\right)$, settling time $\left(T_{s}\right)$, overshoot $\left(\% O_{s}\right)$ and peak for the pitch direction response of a UAV. The results are shown in Fig. 8 and 9 and Fig. 18 represents the responses of the 2DOF-PID controller, PID controller and (NN) controller with and without the adaptive control. During trial and error, the change gamma and alpha values were key to obtain the desired response, which assumes that the gamma is set to 0.5 and alpha is set to 0.1 for all three controllers with and without adaptive control. Figure 8, 9 and 18 demonstrate the simulation approach.

The 2DOF-PID controller is applied with and without the adaptive controller for the UAV pitch control system and the result is shown in Fig. 8. It displays a comparison of the pulse generator response for the 2DOF-PID controller with and without the adaptive controller. In addition, the reference model response in red is the desired response, which is set to 1 . The $2 \mathrm{DOF}-$ PID controller with adaptive control displays in green and the 2DOF-PID controller without the adaptive control displays in blue. In contrast, the plant response of the 2DOF-PID controller with an adaptive control display with a lesser overshoot, which is compared to the 2DOF-PID controller without adaptive control. However, the 2DOF-PID controller with adaptive control demonstrates a better and similar response to the reference model response that is due to the speed of the learning rate that adapts to the changes in the plant response, which improves over time in 60 seconds. Whereas, the theta parameter $(\theta)$ is the adjustable parameter output response of the gamma value, which helps to alter the parameter of the controller to direct the plant response. In addition, the observation of the system's effect with an applied disturbance with producing a learning curve that minimizes the error between the outputs of the plant and the reference model shows the plant is improving during simulation due to fast-changing theta $(\theta)$. The key for the adaptive mechanism is to assist and direct the plant model response to follow the reference model response with a positive gamma value, which is set to 0.5 . The alpha value is greater than 0 , whereas, the alpha is 0.1 .

For the pitch direction response of a UAV, the performance characteristics of UAV are measured and obtained in terms of rise time $\left(T_{r}\right)$, settling time $\left(T_{s}\right)$, overshoot $\left(\% O_{S}\right)$ and peak. Whereas, the desired set point of the pitch amplitude/direction is set to 1 . In this performance analysis, the 2DOF-PID controller is applied with and without the adaptive control to produce output responses with the effect of the disturbance being applied for the UAV pitch control system. The results are shown in Table 1 . The results clearly denote that the 2DOF-PID controller with adaptive control gave a better response than the 2DOFPID controller without adaptive control. As illustrated in Table 1: The Performance Characteristics of UAV, the simulation results reveal that the 2DOF-PID controller with the adaptive control has a smaller rise time $\left(T_{r}\right)$, settling time $\left(T_{s}\right)$ and overshoot $\left(\% O_{S}\right)$ than the 2DOF-PID controller without adaptive control. In comparison, the 2DOF-PID controller with the adaptive control peak value is roughly closer to the desired setpoint compared to the 2DOF-PID controller without the adaptive control. Moreover, the peak value is equivalent to the desired set point.

The PID controller is applied with and without the adaptive controller for the UAV pitch control system and the result is shown in Fig. 9. It displays a pulse generator response comparison of the PID controller with and without the adaptive controller. In addition, the reference model response in red is the desired response, which is set to 1 . The PID controller with adaptive control displays in orange and the PID controller without the adaptive control displays in blue. However, both cases with and without adaptive control did not have sufficient time to reach the desired set point. Whereas, the response was fast and contains a very small overshoot. In contrast, the plant response output of the PID controller with adaptive control displays the response is moving further away and decreasing over time in 60 seconds from the desired response output of the reference model compared to the PID controller without adaptive control. Additionally, the system's effect with an applied disturbance shows that the disturbance drives the plant away from its desired behavior output response of the reference model. Within 60 seconds, the performance are summarized in Table 2.

Table 1: Comparison of UAV performance characteristics

\begin{tabular}{|c|c|c|c|c|}
\hline & $T_{r}(\mathrm{sec})$ & $T_{s}(\mathrm{sec})$ & $O_{S}(\%)$ & Peak \\
\hline \multicolumn{5}{|c|}{ 2DOF-PID without adaptive control } \\
\hline Response & 0.992 & 1.840 & 89.69 & 1.009 \\
\hline \multicolumn{5}{|c|}{ 2DOF-PID with adaptive control } \\
\hline Response & 0.792 & 1.588 & 55.91 & 1.004 \\
\hline
\end{tabular}

Table 2: Comparison of UAV performance characteristics

\begin{tabular}{|c|c|c|c|c|}
\hline & $T_{r}(\mathrm{sec})$ & $T_{s}(\mathrm{sec})$ & $O_{S}(\%)$ & Peak \\
\hline \multicolumn{5}{|c|}{ PID without adaptive control } \\
\hline Response & 1.540 & 13.343 & 0.38 & $9.962 \mathrm{e}-01$ \\
\hline \multicolumn{5}{|c|}{ PID with adaptive control } \\
\hline Response & 1.235 & 12.113 & 0.5 & $9.995 \mathrm{e}-01$ \\
\hline
\end{tabular}




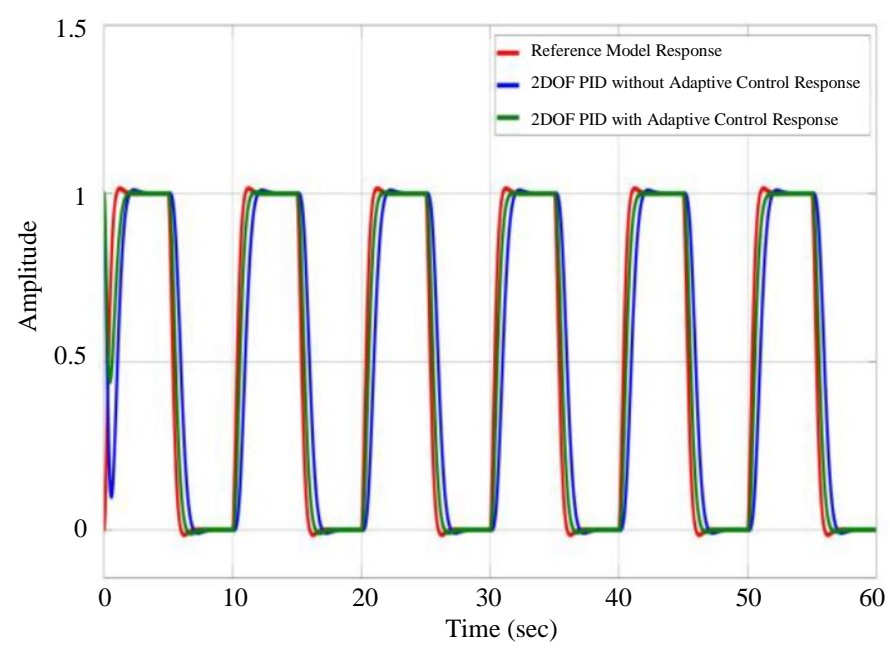

Fig. 8: 2DOF PID Pulse Generator Response with and without Adaptive Control

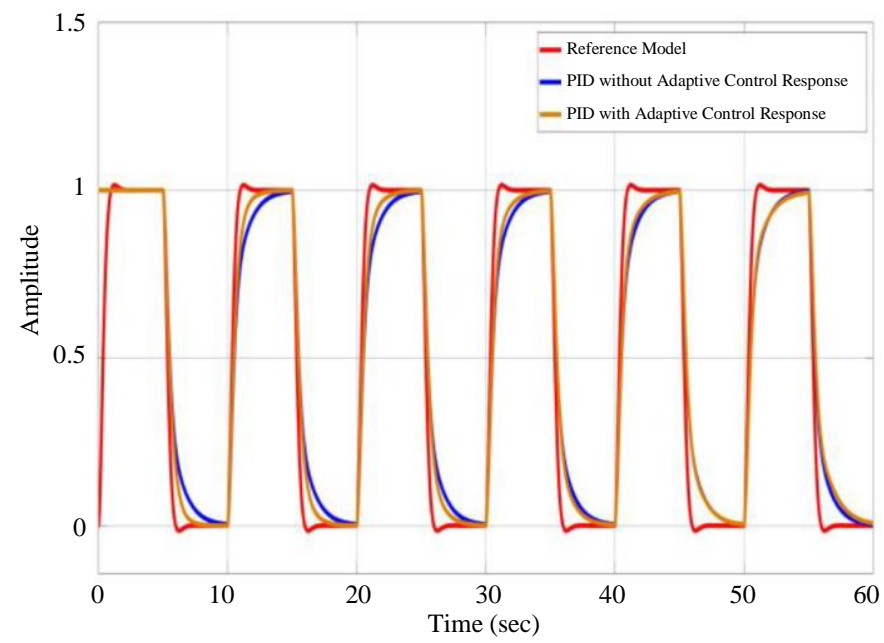

Fig. 9: PID Pulse Generator Response with and without Adaptive Control

The performance characteristics of UAV are measured and obtained in terms of rise time $\left(T_{r}\right)$, settling time $\left(T_{s}\right)$, overshoot $\left(\% O_{s}\right)$ and peak. The desired set point of the pitch amplitude/direction is set to 1 for the pitch direction response of a UAV. The PID controller is applied with and without the adaptive control to produce output responses with the effect of the disturbance being applied for the UAV pitch control system and the results are shown for the performance analysis in Table 2 . The result clearly denotes that the adaptive controlled PID controller gave a better response than the non-adaptive controlled PID controller in Table 2. As illustrated in Table 2, the simulation results reveal that the PID controller with the adaptive control has a smaller rise time $\left(T_{r}\right)$ and settling time $\left(T_{s}\right)$ than the PID controller without adaptive control. In contrast, the overshoot $\left(\% O_{s}\right)$ and the peak value of the adaptive controlled PID controller are greater than the PID controller with no adaptive controller. In addition, the peak value is not a rough equivalent to the desired set point.

For the NN controller, Equation 6 correlates with Fig. 4. It displays the values implemented in the reference model block. The first step in the neural network process is to approximate the behavior of the plant/system identification on the neural network. The main concept of plant/system identification is to obtain a model that can be used for controller design. This is widely used for applications ranging from control system design and signal processing to time series analysis (Suzuki, 2011). In the system identification stage, the goal is to apply the plant identification process to allow the NN to develop and train itself to model the output of the plant that needs to be controlled. Secondly, it is necessary to identify the plant before the controller is trained. The three layers with 10 neurons in the hidden layer are utilized as NN architecture. 

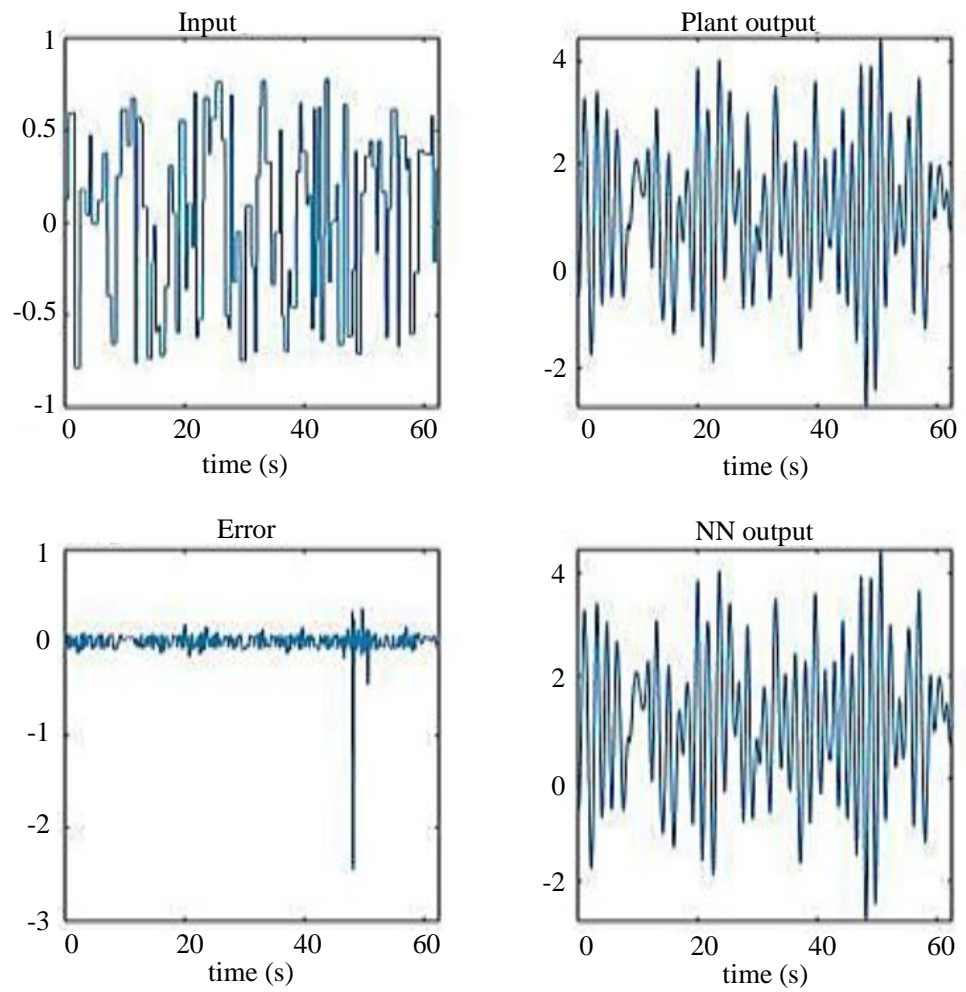

Fig. 10: Testing data
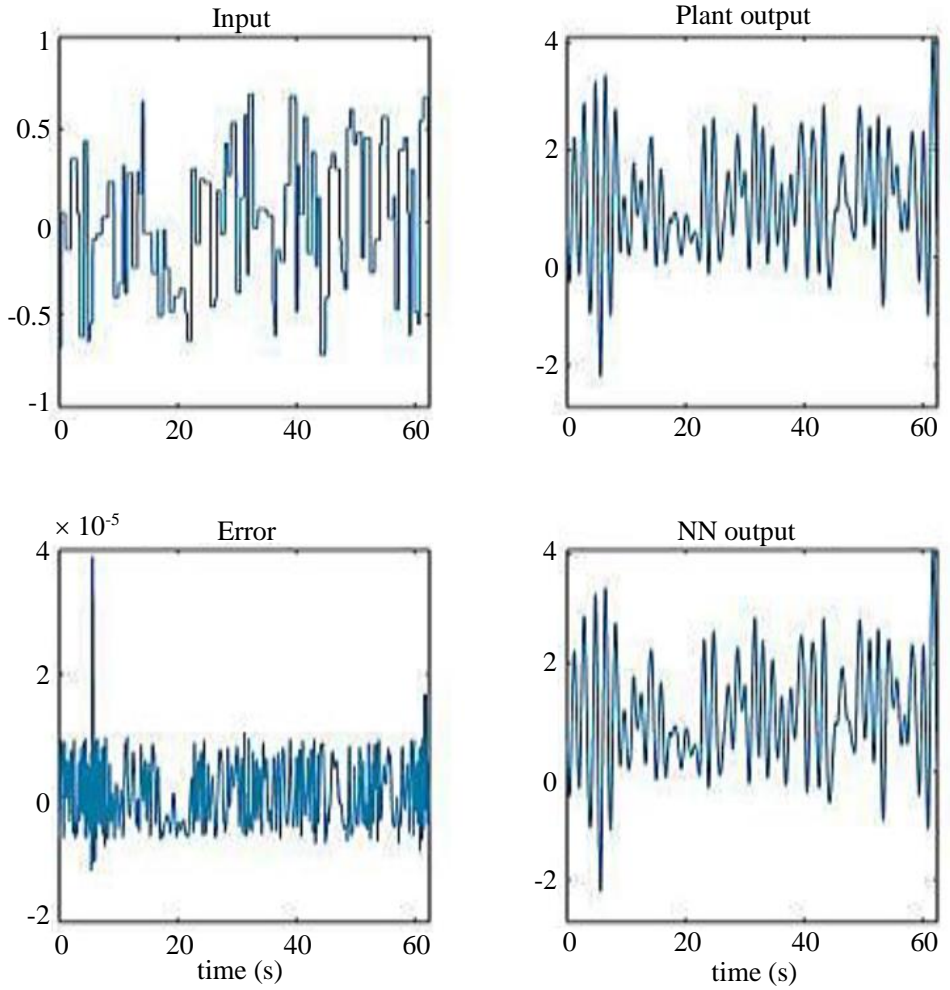

Fig. 11: Validation data 

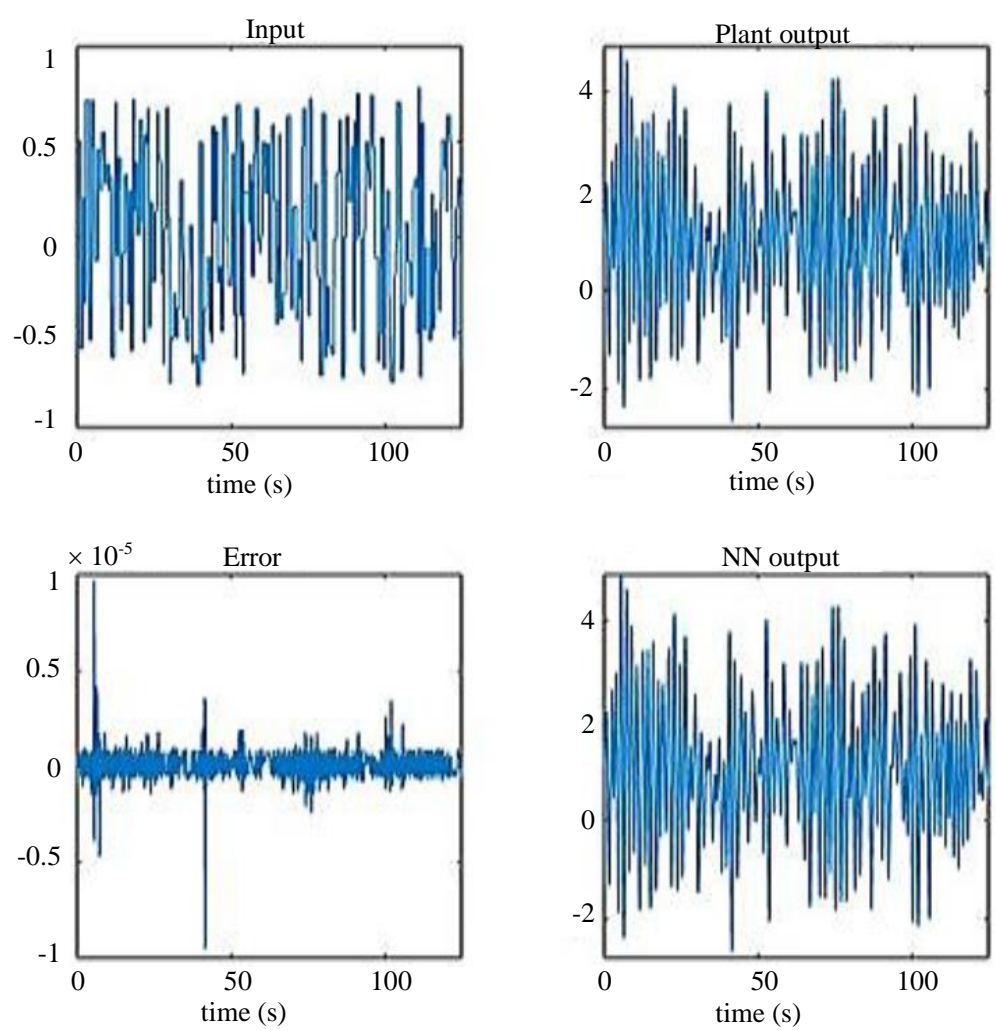

Fig. 12: Training data
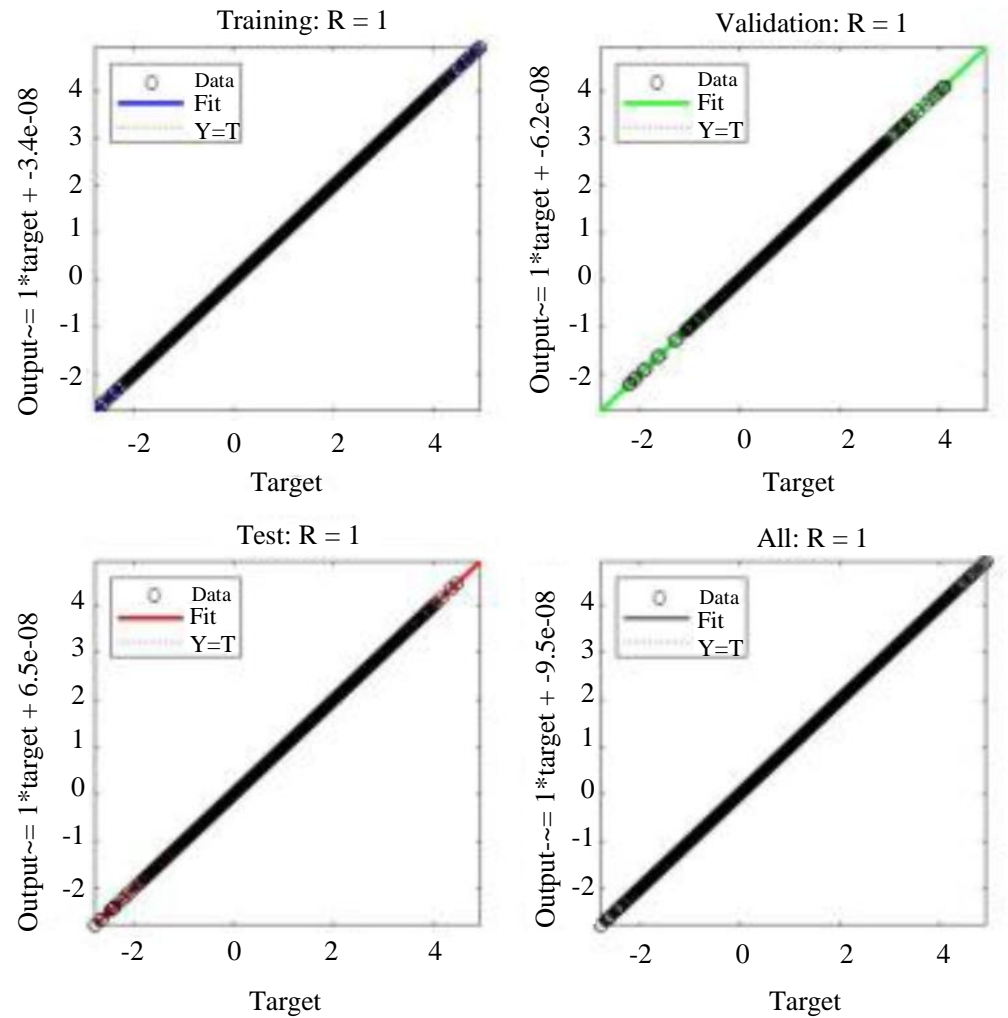

Fig. 13: Training regression 
Two delayed inputs $u(k-1), u(k-2)$ and two delayed outputs $y(k-1), y(k-2)$ with a sampling period of 0.05 of the actual plant are used as input to capture the system dynamics (Eressa et al., 2016). Five thousand (5,000) samples of input/output maximum/minimum with the size of $0.7854 /-$ 0.7854 radians are used for plant neural model training (Eressa et al., 2016).

The next step generates a training data process. After training, the data generation is complete and the network training process begins. During this process, the goal is to identify the plant as the Levenberg-Marquardt (trainlm), which provides a gradient based technique allowing fast error minimization between the actual output of the system and the predicted output of the network (Suzuki, 2011). This demonstrates the validation, training and testing data from the network training process as illustrated in Fig. 10 to 12 . The training, validation and testing data errors should remain very small and the plant's output should be similar to the NN output.

In Fig. 13, the regression data demonstrates the linear regression of targets parallel to the outputs. In Fig. 14, the graph displays the neural network's reported training performance in the plant identification process during training (300 Epochs). Whereas, the training data and testing data follows almost the same behavior. In the mean square graph, the error is very small, while the validation stops decreasing and discontinues the training process. The performance must reveal a small value for practical purposes.

In the control design stage, the next step repeats the exact same process similar to the plant identification in the Model Reference Controller and utilizes training the controller process as illustrated in Fig. 15 and Fig. 16. The MATLAB technique reports the performance is shown in Fig. 15 and Fig. 16 for the (BFGS) quasiNewton backpropagation (trainbfg) (Demuth et al., 1992). The use of the neural network plant model to design/train the controller uses the propagation of the controlling error through the NN model (Suzuki, 2011). Likewise, the training produces the optimal connection weights for the networks by minimizing the errors between $\mathrm{NN}$ output and the plant output over the entire set of samples (Suzuki, 2011). The NN controller is trained to track the reference model given in Fig. 4. Two delayed reference inputs $r(k-1), r(k-2)$ one delayed controller input $C(k-1)$ and two delayed outputs $y(k-1), y(k-2)$ with a sampling period of 0.05 is used as inputs to the NN controller (Eressa et al., 2016). The NN architecture contains three layers with 5 neurons in the input, 5 neurons in the hidden layer and 1 neuron in the output layer. However, 2,000 samples with input/output maximum/minimum size of 4/-4 radians are used for controller training (Eressa et al., 2016). This method will analyze and research the plant itself to observe and identify the behavior that interacts with the neural network controller. This process presents segments of data to the network and trains it for a specific number of iterations. Before training a controller, the plant is identified and the controller is designed to create the plant output to follow the reference model output.

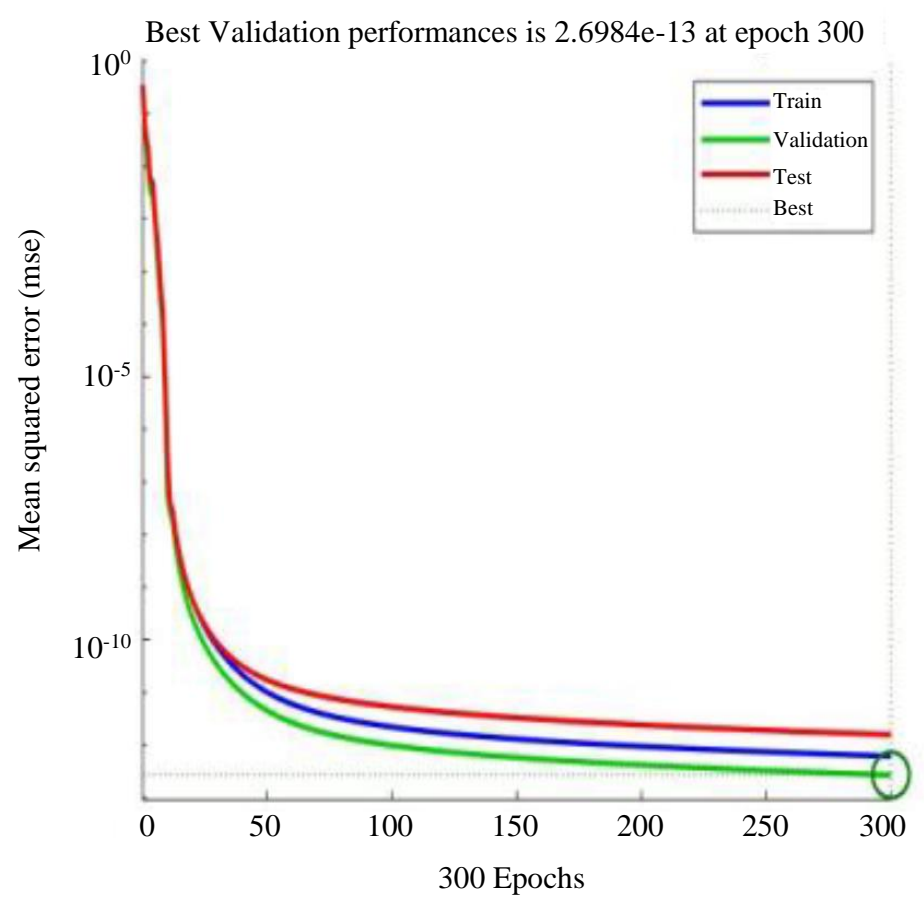

Fig. 14: Mean Square Error Graph 


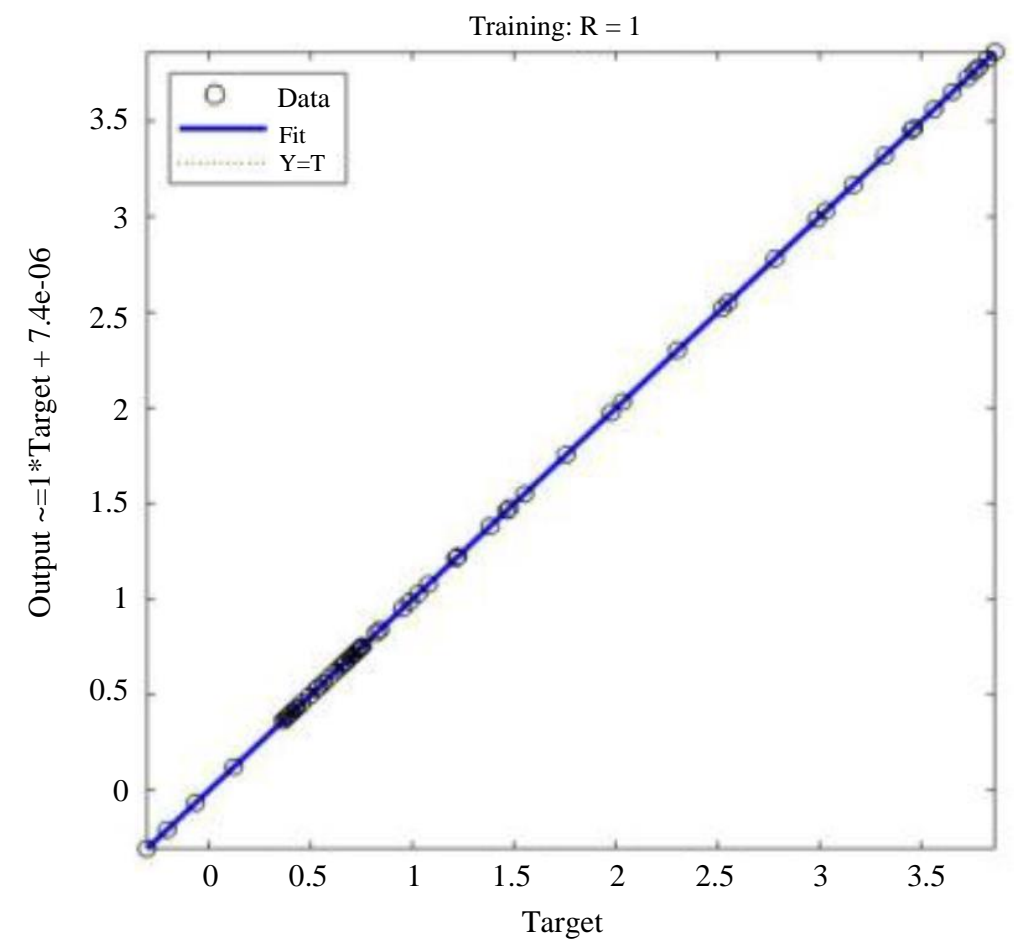

Fig. 15: Neural network training regression

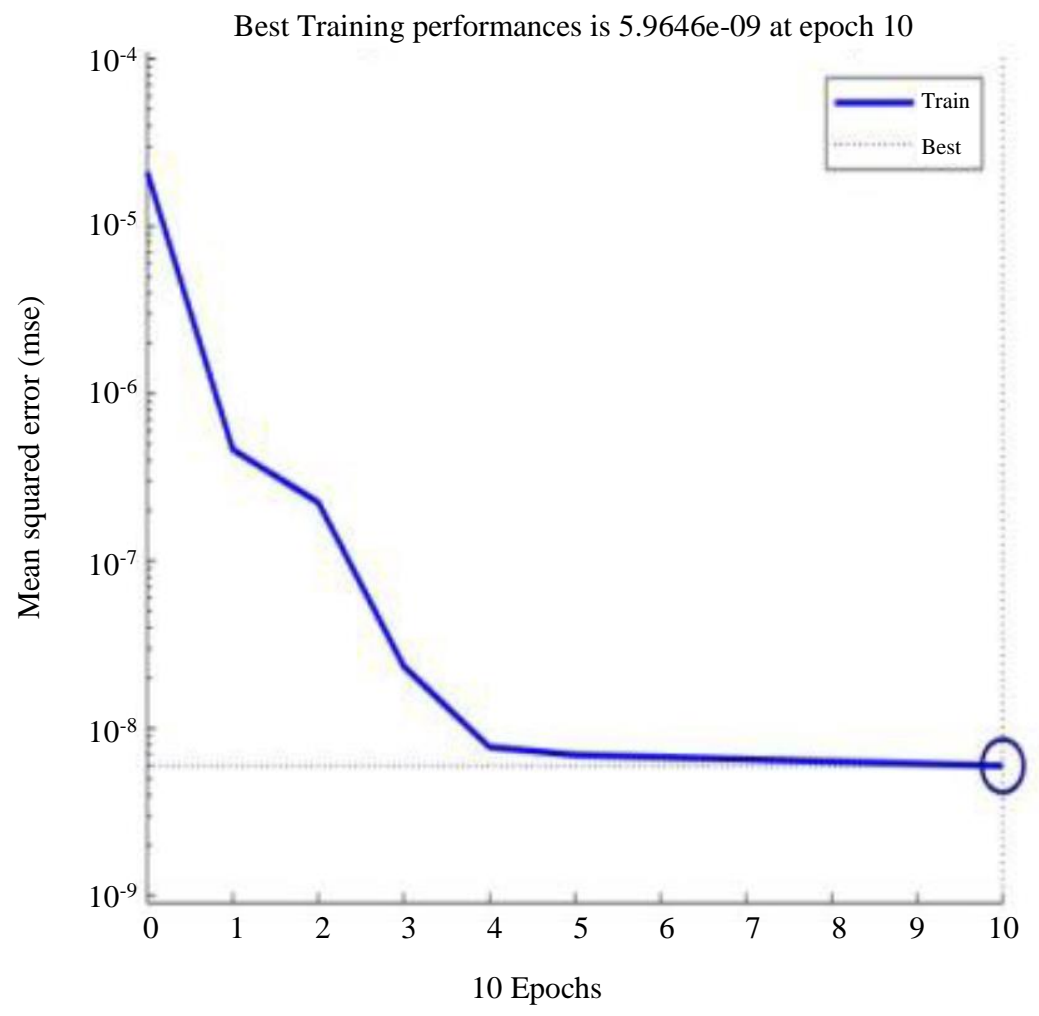

Fig. 16: Neural network training performance 
During trial and error, the NN tracks the reference model's results by setting the considered amount of controller trained samples and controller training epochs in Fig. 17. The axis above the response output of NN follows the response of the reference model and demonstrates the random reference input used for training. The $\mathrm{NN}$ is trained and it controls itself to display an output response described by the reference model. After training, the controller process is complete and the response of the resulting feedback control is demonstrated in Fig. 17. The end result can produce a healthy and functioning system using the NN.

In Fig. 18, it displays a pulse generator response comparison of the NN controller with and without the adaptive controller. In addition, the reference model response in red is the desired response, which is set to 1. The NN with adaptive control displays in purple and the NN without the adaptive control displays in blue. However, the NN controller with adaptive control demonstrates that the speed of the learning rate adapts to the changes in the plant response, which improves over time in 60 seconds. It has a similar response to the reference model. The key role for the adaptive mechanism is to assist and direct the plant model response to track the reference model response with a positive gamma value and alpha value, which is set to 0.5 and 0.1 .

In this performance analysis, the NN controller is applied with and without the adaptive control to produce output responses with the effect of the disturbance being applied for the UAV pitch control system and the results are shown in Table 3. For the performance characteristics of UAV, the simulation results reveal the NN controller without the adaptive control has a higher rise time $\left(T_{r}\right)$, settling time $\left(T_{s}\right)$ and overshoot $\left(\% O_{s}\right)$, because of the effect of the disturbance that is applied to the plant/system model compared to the NN controller with adaptive control. In comparison, the NN controller with the adaptive control peak value is approximately very close to the desired set-point compared to the NN controller without the adaptive control. In Table 3, the performance clearly indicates that the adaptivecontrolled NN controller gives a better response than the NN controller without adaptive control. With the same gamma value and alpha value, it displays that the Neural Network is trained and it controls itself to display an output response described by the reference model. After training, the controller process is complete and the response of the resulting feedback control is demonstrated in Fig. 18. This NN controller is developed, in such a way, to generate the plant output to track the output of the reference model. Moreover, the plant response output and reference model output are very close. If the plant model is not accurate, it can affect the controller's training.

Table 3: Comparison of UAV performance characteristics

\begin{tabular}{|c|c|c|c|c|}
\hline & $T_{r}(\mathrm{sec})$ & $T_{s}(\mathrm{sec})$ & $O_{s}(\%)$ & Peak \\
\hline \multicolumn{5}{|c|}{ NN without Adaptive Control } \\
\hline Response & 0.821 & 1.473 & 55.95 & 1.005 \\
\hline \multicolumn{5}{|c|}{ NN with Adaptive control } \\
\hline Response & 0.604 & 1.015 & 13.32 & 1.001 \\
\hline
\end{tabular}
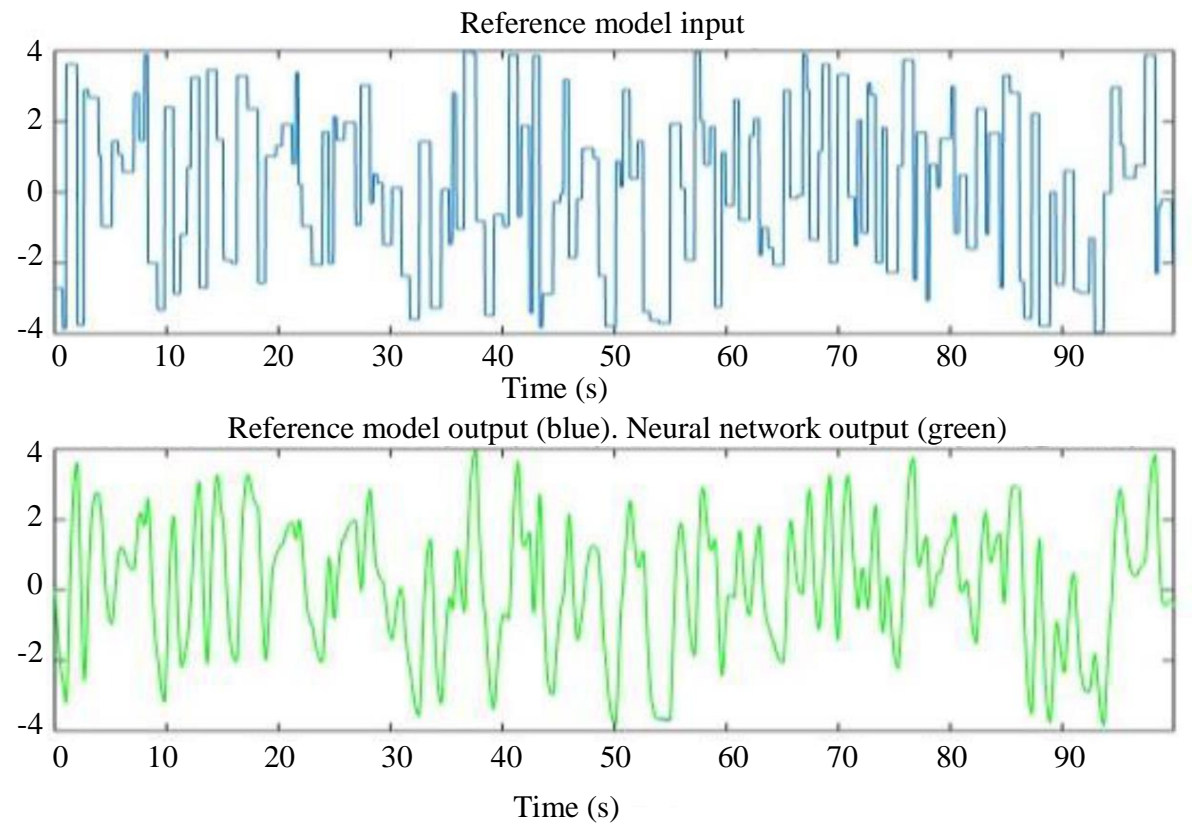

Fig. 17: Plant response for NN model reference control 


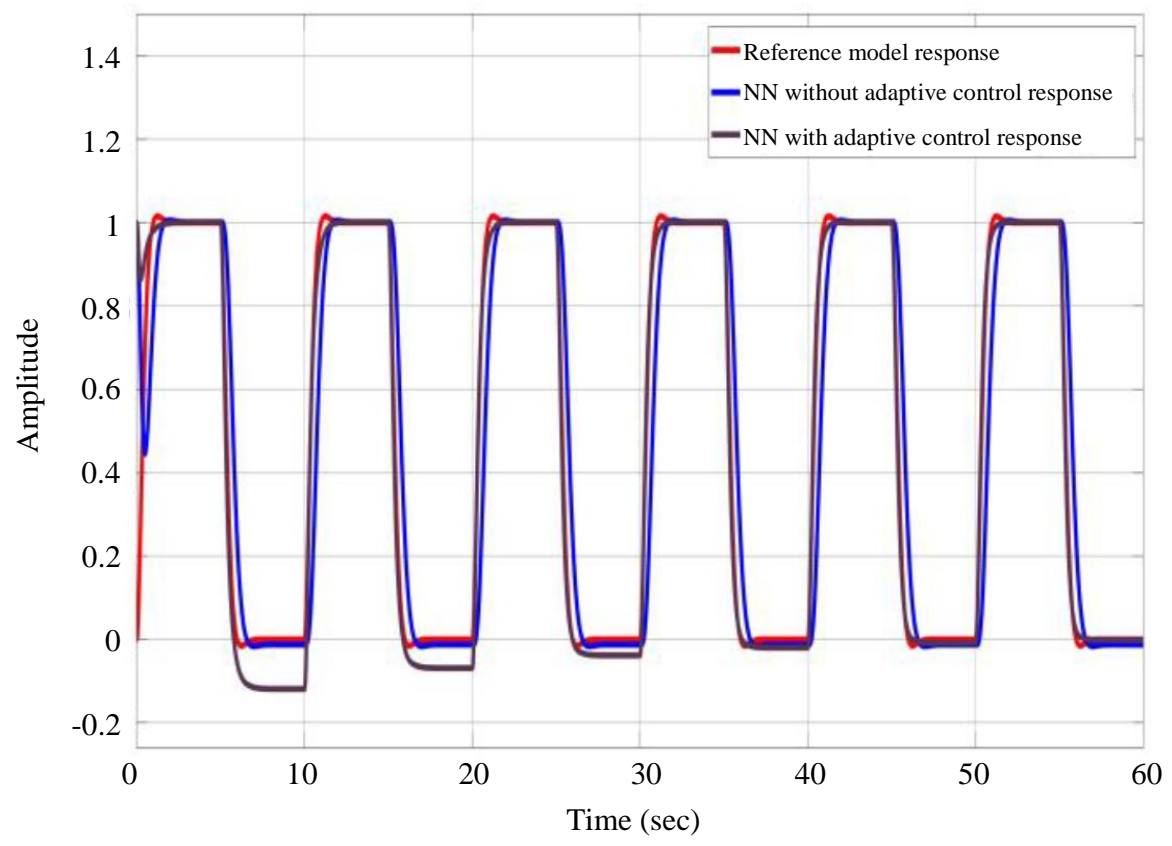

Fig. 18: NN pulse generator response comparison of adaptive control

\section{Conclusion and Future Work}

In conclusion, the aim of this scope of research will generate a flexible, robust and efficient model of a control system that offers greater stability and reduces the impacts of disturbances. In addition, the controller's efficiency was evaluated through simulations by using the software, MATLAB, which show enhanced speed and stability. Thus, the robust controller identifies and decreases the unpredictable impacts of disturbances and attains better system behavior. The reason for the creation of a robust control system with a controller and adaptive control will enhance navigation efficiency and sensing for military, recognition and monitoring applications. In developing a controller for these aircraft, there are numerous sources for the uncertainty that corresponds to disturbances in the system. The PID controller, 2DOF-PID controller and NN controller with adaptive control were used to analyze high-performance in the presence of such disturbance. A system disturbance is implemented to test and assess performance characteristic analysis. Furthermore, disturbances can result in a system's instability and unpredictable behaviors. Therefore, the UAV responds to modeling errors and disturbances with attaining the desired state, which depends on the strength of the control system used. The validation and observation of the $\mathrm{NN}$ controller show, that a NN controller with the adaptive control response of a positive gamma value with an alpha value has high-performance tracking with increased robustness in the presence of parametric uncertainties than the 2DOF-PID and PID controllers. Moreover, the NN controller has a better overtime response that follows the desired response of the reference model more precisely than the 2DOF-PID and PID controllers, which is demonstrated in Table 3 for the performance characteristics of UAV results and simulation results in Fig. 18. From this observation, the $\mathrm{NN}$ is appropriately learning from a set of training patterns by observing the scheme. System identification utilizes a NN to capture system dynamics, which trains the Neural Network to behave as a controller. The adaptive controlled NN controller demonstrates that it improves the stability and response speed of the system than the NN without the adaptive control response. Consequently, the limitations are the fixed PID and 2DOF-PID terms with the guessing method for the gamma and alpha values. It retrains the NN controller to direct the plant model with a higher set point amplitude to obtain the desired response or to increase the gamma and alpha values. Moreover, the high order Modified MIT Rule adaptive control performance illustrates the adaptation in the change of the plant's output that stabilizes the system by the controller component into the system. Similarly, the implementation of a gamma value was used to produce a learning curve to minimize the error between the plant and the reference model outputs that are influenced by the adaptation gain. Thus, the alpha value removes the zero-division error when the system's sensitivity derivative is low. 
For future works, the experimental validation plan will require the purchase of new equipment to test the development of a controller. Further study, testing and research will continue to explore the application of a robust controller with a neural network estimator for development. In addition, the plan will apply and compare the NN controller, Neural-PID controller and PID controller with adaptive control with the use of the second order system MIT Rule. Hence, this plan of study will understand the attributes of the second order system MIT Rule for the MRAC. This investigation will determine the performance by observing the system's behavior to identify and reduce the unpredictable effects of disturbance in a UAV control system. This research continues as a work in progress for the neural network to train and control itself to display a response with the adaptive control prescribed by the reference model with an applied disturbance.

\section{Acknowledgement}

I would like to express my gratitude to Dr. Sun Yi, for his encouragement and constructive critique of this research. I would like to express my sincere appreciation to the College of Engineering and TECHLAV for the support of my academic pursuits at North Carolina A\&T State University.

\section{Author's Contributions}

Mackenzie Matthews: Collected the data, conducted the simulations and obtained the results.

Sun Yi: Developed the theoretical framework and supervised the research.

\section{Ethics}

This article is an original research paper. There are no ethical issues that may arise after the publication of this manuscript.

\section{References}

Aamir, M., 2013. On replacing PID controller with ANN controller for DC motor position control.

Alfaro, V. and R. Vilanova, 2016. Two-Degree-of-Freedom PID Controllers Structures. In: Model-Reference Robust Tuning of PID Controllers, Springer, Cham, ISBN-13: 978-3-319-28211-4, pp: 7-19.

Armah, S.K., 2015. Adaptive control for autonomous navigation of mobile robots considering time delay and uncertainty. $\mathrm{PhD}$ Thesis, North Carolina Agricultural and Technical State University, Greensboro, North Carolina, United States.

Armah, S.K., 2018. Adaptive control for quadrotor uavs considering time delay: study with flight payload. Robot. Automat. Eng. J., 2: 1-13.

DOI: $10.19080 /$ RAEJ.2018.02.555598
Cuong, N.D., N. Van Lanh and D. Van Huyen, 2013. Design of MRAS-based adaptive control systems. Proceedings of the International Conference on Control, Automation and Information Sciences, Nov. 25-28, IEEE Xplore Press, Nha Trang, Vietnam. DOI: 10.1109/ICCAIS.2013.6720534

Demuth, H., M. Beale and M. Hagan, 1992. Neural network toolbox. For use with MATLAB. The MathWorks Inc.

Dydek, Z.T., A.M. Annaswamy and E. Lavretsky, 2013. Adaptive control of quadrotor UAVs: A design trade study with flight evaluations. IEEE Tran. Control Syst. Technol., 21: 1400-1406.

DOI: 10.1109/TCST.2012.2200104

Eressa, M.R., Z. Danchen and H. Min, 2016. PID and neural net controller performance comparsion in UAV pitch attitude control. Proceedings of the IEEE International Conference on Systems, Man and Cybernetics, Oct. 9-12, IEEE Xplore Press, Budapest, Hungary. DOI: $10.1109 /$ smc.2016.7844333

Ghaffar, A.A. and T. Richardson, 2015. Model reference adaptive control and LQR control for quadrotor with parametric uncertainties. Int. J. Mech. Aerospace Industrial Mechatronic Manufact. Eng., 9: 244-250.

Goel, A., R. Kumar and S. Narayan, 2016. Design of MRAC augmented with PID controller using genetic algorithm. Proceedings of the IEEE 1st International Conference on Power Electronics, Intelligent Control and Energy Systems, Jul. 4-6, IEEE Xplore Press, Delhi, India. DOI: 10.1109/ICPEICES.2016.7853149

Horsman, G., 2016. Unmanned aerial vehicles: A preliminary analysis of forensic challenges. Digital Invest., 16: 1-11. DOI: 10.1016/j.diin.2015.11.002

Ioannou, P. and S. Baldi, 2010. Robust adaptive control. In: The Control Systems Handbook, Levine, W.S. (Ed.), CRC Press, ISBN-10: 1420073648, pp: 1-22.

Jain, P. and M. Nigam, 2013. Design of a model reference adaptive controller using modified MIT rule for a second order system. Adv. Electronic Electric Eng., 3: 477-484.

Janert, P.K., 2013. Feedback Control for Computer Systems: Introducing Control Theory to Enterprise Programmers. 1st Edn., O'Reilly Media, Inc., ISBN-10: 1449362656, pp: 336.

Kasparian, V. and C. Batur, 1998. Model reference based neural network adaptive controller. ISA Tran., 37: 21-39. DOI: 10.1016/S0019-0578(98)00002-0

Matthews, M. and S. Yi, 2019. Model reference adaptive control and neural network based control of altitude of unmanned aerial vehicles. IEEE SoutheastCon.

Palm, W.J., 2014. System Dynamics. 3rd Edn., McGraw-Hill Science, ISBN-10: 0073398063. 
Suzuki, K., 2011. System Identification of NN-Based Model Reference Control of Ruav During Hover. In: Artificial Neural Networks-Industrial and Control Engineering Applications, Suzuki, K. (Ed.), BoDBooks on Demand, North-Holland, ISBN-10: 9533072202, pp: 395-420.

Sarhan, A. and S. Qin, 2016. Adaptive PID control of UAV altitude dynamics based on parameter optimization with fuzzy inference. Int. J. Model. Optimizat., 6: 246-251.

DOI: 10.7763/IJMO.2016.V6.534

Socas, R., S. Dormido and R. Dormido, 2015. Eventbased control strategy for the guidance of the aerosonde UAV. Proceedings of the European Conference on Mobile Robots, Sept. 2-4, IEEE Xplore Press, Lincoln, UK.

DOI: 10.1109/ECMR.2015.7324213
Swarnkar, P., J. Shailendra Kumar and R. Nema, 2011a Comparative analysis of MIT rule and lyapunov rule in model reference adaptive control scheme.

Swarnkar, P., S. Jain and R. Nema, 2011b. Effect of adaptation gain in model reference adaptive controlled second order system. Eng. Technol. Applied Sci. Res., 1: 70-75.

Swathi, M. and P. Ramesh, 2017. Modeling and analysis of model reference adaptive control by using MIT and modified MIT rule for speed control of dc motor. Proceedings of the IEEE 7th International Advance Computing Conference, Jan. 5-7, IEEE Xplore Press, Hyderabad, India. DOI: 10.1109/IACC.2017.0105 